\title{
Calculation of Potential Energy for Fe Atom around Entrance of $(10,0)$ Carbon Nanotube
}

\author{
Yohei Kudo $^{1}$, Takahisa Hira ${ }^{1}$, Soh Ishii $^{1}$, Tsuguo Morisato ${ }^{2}$ and Kaoru Ohno ${ }^{1}$ \\ ${ }^{1}$ Department of Physics, Graduate School of Engineering, Yokohama National University, Yokohama 240-8501, Japan \\ ${ }^{2}$ Accelrys K.K., Nishishinbashi TS Bldg. 11F, Tokyo 105-0003, Japan
}

The structure of carbon nanotubes constitutes graphene sheets that are rolled to form cylinders with extremely small diameters. It is interesting to investigate the interaction between single-walled carbon nanotubes and an Fe atom because such nanotubes are fabricated with the aid of metal catalysts ( $\mathrm{Fe}, \mathrm{Co}$, etc). Using an ab initio program $\mathrm{DMol}^{3}$, we have calculated the total energy of a system of (10,0) nanotube incorporated with an Fe atom. We have determined the most stable position of Fe near the entrance of the nanotube and the potential map for that position. [doi:10.2320/matertrans.47.2462]

(Received July 3, 2006; Accepted August 17, 2006; Published October 15, 2006)

Keywords: single-walled carbon nanotube, first-principles, total energy, catalyst, iron

\section{Introduction}

Fullerene, carbon's third allotrope, ${ }^{1)}$ multi-walled carbon nanotubes $^{2)}$ and single-walled carbon nanotubes (SWNTs) ${ }^{3)}$ were successively discovered in 1985, 1991, and 1993, respectively. Since then, the study of carbon nanotubes (CNTs) has progressed rapidly. Today, SWNTs are expected to be widely applied to nano-devices. Recently, two first principles studies involving the insertion of foreign atoms and molecules into the SWNTs were carried out. One was a first principles molecular dynamics simulation to insert sodium or potassium atom into SWNTs through the tube surface which clarified that encapsulation was possible. ${ }^{4)}$ The other involved the calculation of the electronic structure calculation of carbon peapod, $\left(\mathrm{C}_{60}\right)$-encapsulated SWNT. ${ }^{5}$ ) With regard to carbon peapod, it was experimentally observed that fullerenes inside the peapod coalesced into each other and formed stable zeppelin-like carbon molecules at a suitably high kinetic energy. ${ }^{6}$ ) One way to synthesize SWNTs is the arc discharge method, in which the mixture of metal and carbon is evaporated in an inert gas atmosphere. Fe is used as a metal catalyst in this manner. ${ }^{7)}$ By this method, cage or tube structures are formed with carbon atoms, and SWNTs are created. Therefore, we can consider that there is an interesting relationship between SWNTs and an Fe atom; however, to the best of our knowledge, the behavior of an $\mathrm{Fe}$ atom in SWNTs has not been investigated theoretically. In this study, we analyze the most stable position and the potential curve of an Fe atom in an SWNT by using a first principles method.

In this study, we consider a $(10,0)$ SWNT composed of 80 carbon atoms. We select points $A \sim G$ near the entrance of the SWNT, as shown in Fig. 1. From these points, we draw perpendicular lines to the central axis of the optimized SWNT. We then place an Fe atom on the perpendicular lines from the points $A \sim D$ and $E \sim G$ to the central axis, as shown in Figs. 2 and 3, respectively. We also select points $H \sim K$ on the central axis, shown in Fig. 4, and place an $\mathrm{Fe}$ atom on each of these points. At each position, we calculate the total energy of the system to find the most stable Fe position.

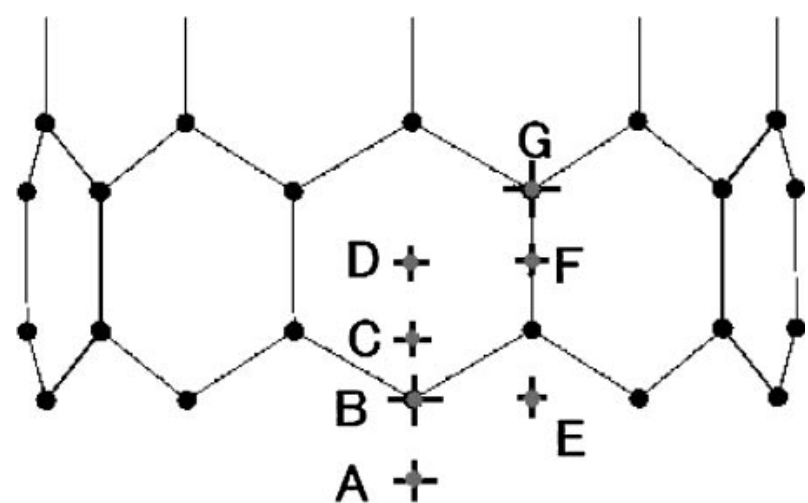

Fig. 1 The starting locations of the perpendicular lines on which total energy is calculated.

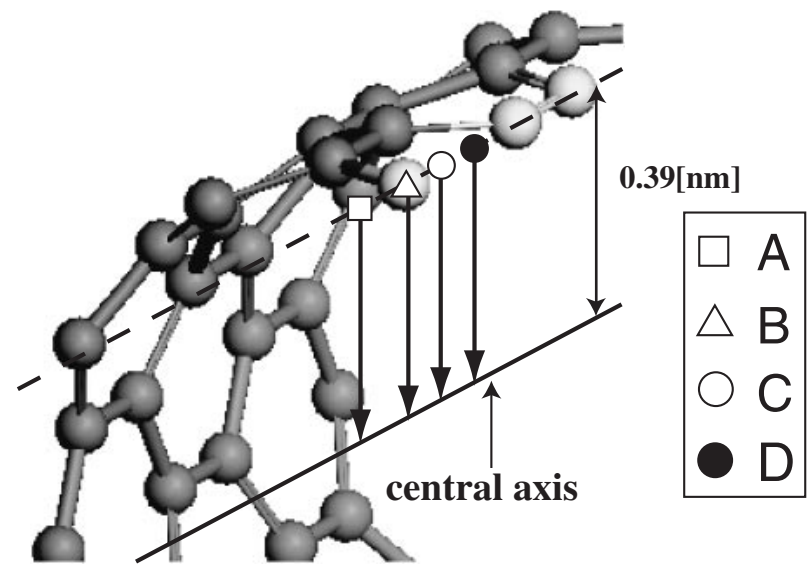

Fig. 2 The perpendicular lines starting from points $A \sim D$ directing to the center axis.

\section{Methodology}

For the first principles calculation, we adopt the program package $\mathrm{DMol}^{3}$ in Materials Studio (version 3.2) of Accelrys Inc. ${ }^{8-10)}$ This is an all-electron method that uses numerical orbitals for the basis functions, each of which correspond to a localized atomic orbital (AO). Since we use only a localized 


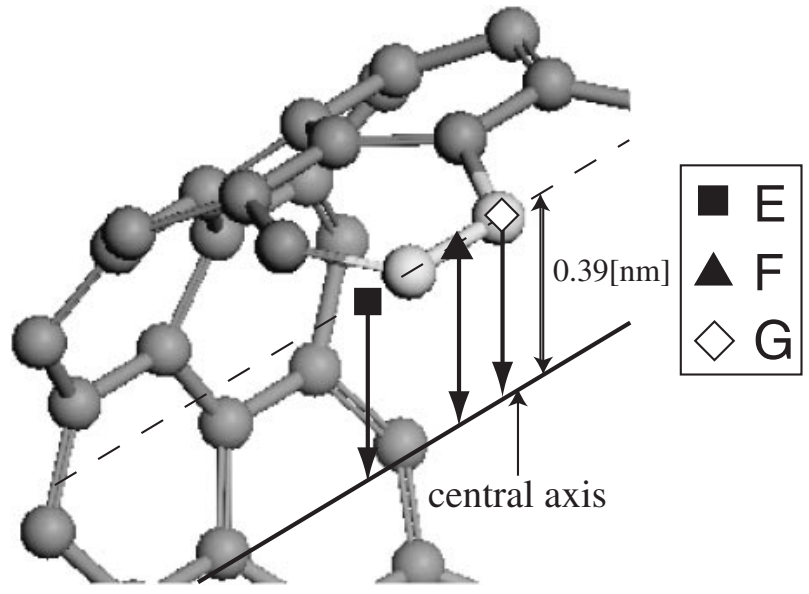

Fig. 3 The perpendicular lines starting from points $E \sim G$ directing to the center axis.

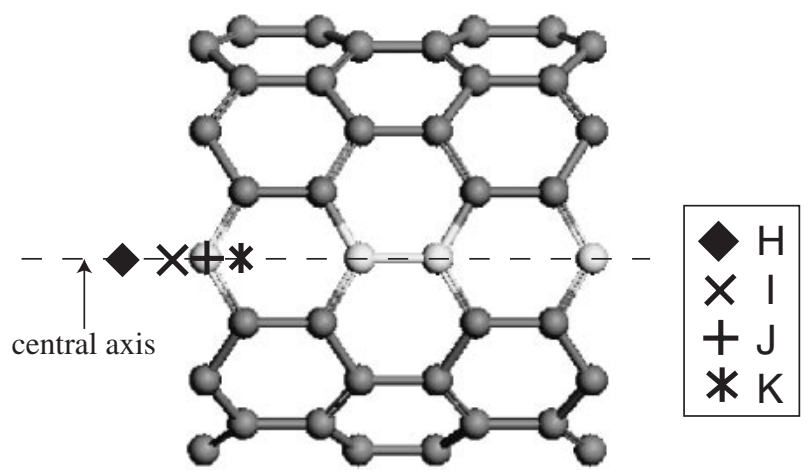

Fig. 4 Points $H \sim K$ on the center axis.

basis in this calculation, the cell is nonperiodic. We use the double numerical plus d-function (DND). It provides a reasonable accuracy at a modest computational cost. We use the Perdew-Wang correlation (PWC ${ }^{11)}$ based on the local spin density approximation (LSDA) of the density functional theory (DFT) for the calculation. In the calculation, we use the smearing of electron occupancy to obtain a good convergence. We gradually reduce the value of the smearing parameter and perform the calculation again. We repeat this procedure until the value of the smearing parameter reduces to zero. We then analyze the behavior of the potential curve for an $\mathrm{Fe}$ atom.

\subsection{Test calculation for ferrocene}

Ferrocene $\left(\mathrm{Fe}\left(\mathrm{C}_{5} \mathrm{H}_{5}\right)_{2}\right)$ consists of an iron atom, ten carbon atoms, and ten hydrogen atoms. We optimized ferrocene by $D \mathrm{Mol}^{3}$ for the test calculation. We also optimized it by Gaussian03. ${ }^{12)}$ Figure 5 represents ferrocene optimized by $\mathrm{DMol}^{3}$, and Figure 6 represents that by Gaussian03. A comparison between the results revealed that the difference between the $\mathrm{C}-\mathrm{C}$ bond length by Gaussian 03 and that by $\mathrm{DMol}^{3}$ was about $0.019 \%$. With regard to the $\mathrm{C}-\mathrm{Fe}$ and $\mathrm{C}-\mathrm{H}$ bonds, the result by $\mathrm{DMol}^{3}$ was about 1.10 and $0.0029 \%$ greater than that by Gaussian03. Further, the result by $D M o l^{3}$, in comparison to that by Gaussian03, for the angle formed by a $\mathrm{C}-\mathrm{C}$ bond and a $\mathrm{C}-\mathrm{H}$ bond and that formed by a $\mathrm{C}-\mathrm{H}$ bond and $\mathrm{C}-\mathrm{Fe}$ bond was $0.016 \%$ greater and $0.32 \%$ smaller than that by Gaussian03. Moreover, the angle formed by two

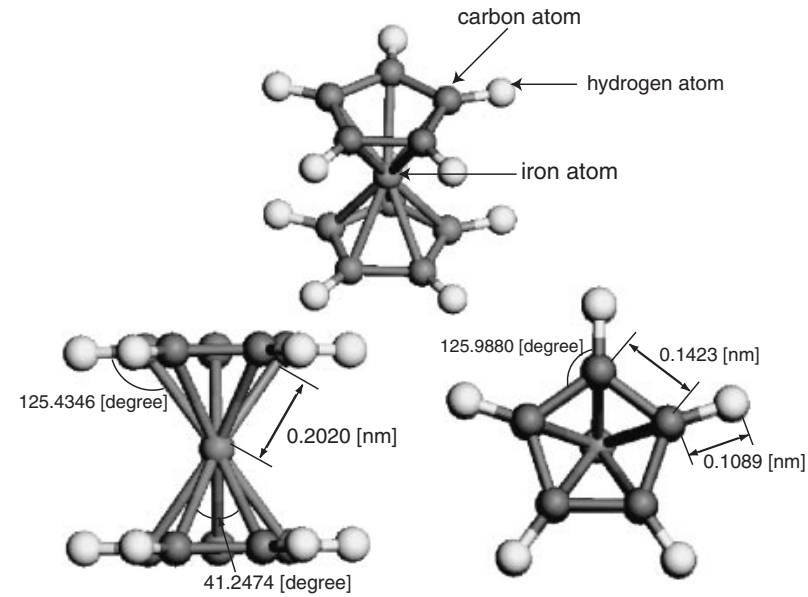

Fig. 5 Ferrocene optimized by $\mathrm{DMol}^{3}$.

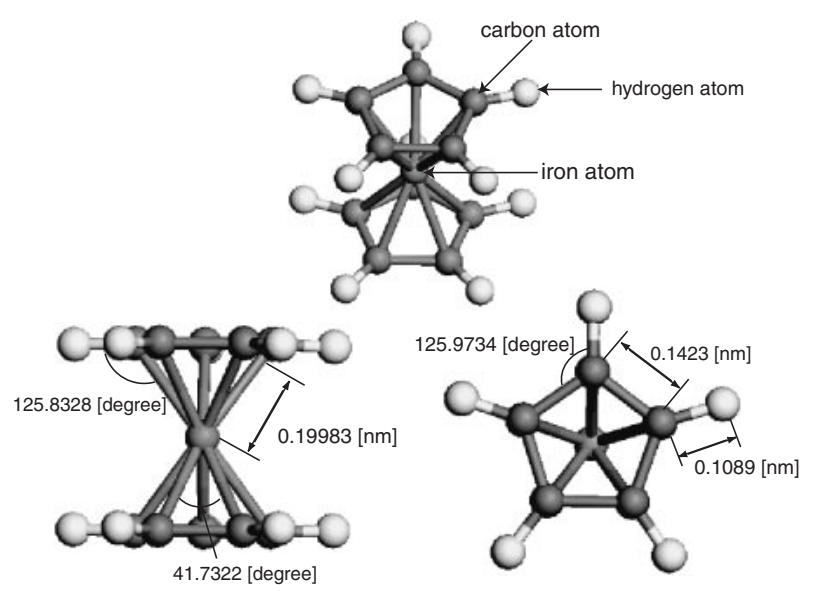

Fig. 6 Ferrocene optimized by Gaussian 03 .

nearest $\mathrm{C}$-Fe bonds is 1.16 percent smaller. Based on these facts, it is evident that the result by $\mathrm{DMol}^{3}$ agreed well with that by Gaussian03.

\section{Results and Discussion}

We now present the results of the calculated total energy. Here, all the energies have relative values.

\subsection{Points $A \sim D$}

The result calculated on the perpendicular lines from points $A \sim D$ to the central axis, shown in Fig. 2, is plotted in Fig. 7. In Fig. 7, the abscissa is the distance from the surface of the SWNT. We carry out calculations based on the position where an $\mathrm{Fe}$ atom is not too close to the $\mathrm{C}$ atoms.

This result suggests that the total energy of the system increases from point $A_{\perp}$ to $D_{\perp}$. (Here $A_{\perp}$ implies the result calculated on the perpendicular line from point $A$. The same meanings hold for the other symbols.) Therefore, the potential energy of an $\mathrm{Fe}$ atom increases and the $\mathrm{Fe}$ atom becomes unstable when it enters deeper into the tube. This suggests that it is difficult for an Fe atom to enter the SWNT. Furthermore, except for the position near the surface of SWNT, an Fe atom tends to be unstable when it approaches the central axis. 


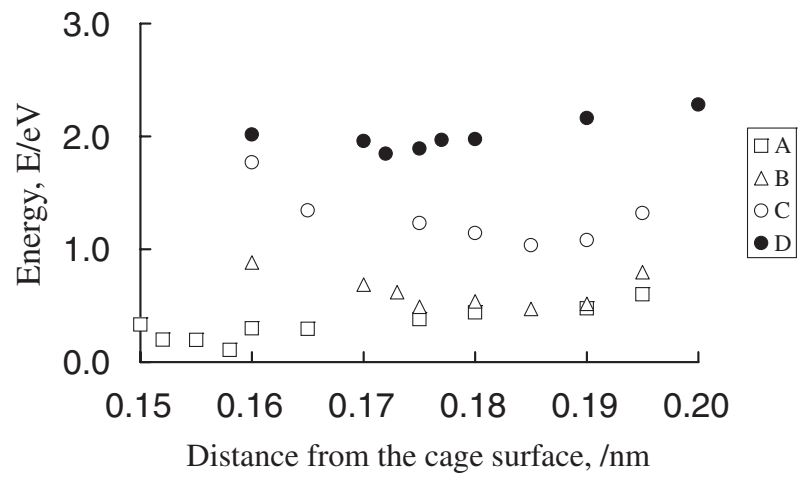

Fig. 7 Calculated total energy on the perpendicular lines starting from points $A \sim D$

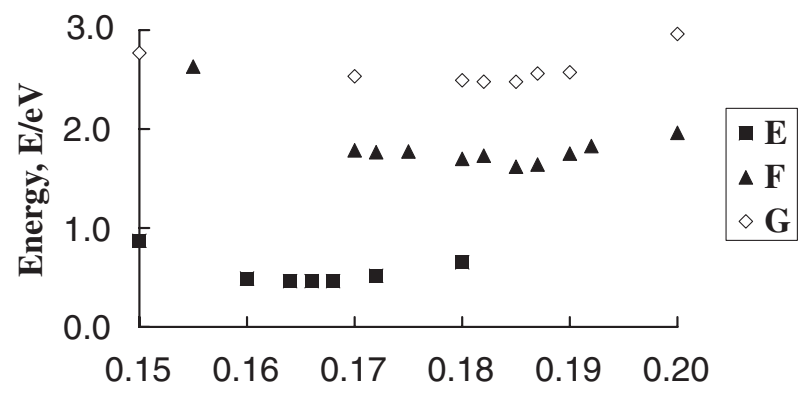

Distance from the nanotube surface, $/ \mathrm{nm}$

Fig. 8 Calculated total energy on the perpendicular lines starting from points $E \sim G$

\subsection{Points $E \sim G$}

The result calculated on the perpendicular lines from points $E \sim G$ to the central axis, shown in Fig. 3, is plotted in Fig. 8.

The total energy of the system increases from point $E_{\perp}$ to $G_{\perp}$, and when an $\mathrm{Fe}$ atom approaches the central axis, it becomes unstable. This tendency is common to that in the case of points $A_{\perp} \sim D_{\perp}$. Therefore, the potential energy of an $\mathrm{Fe}$ atom increases and the atom becomes unstable when it enters deeper into the tube.

\subsection{Points $H \sim K$}

The result of the total energy at points $H \sim K$ on the central axis, shown in Fig. 4, is plotted in Fig. 9.

According to this result, when an $\mathrm{Fe}$ atom moves away from the SWNT entrance, it becomes unstable. We can see that the differences between points $I \sim K$ are smaller than those between lines $A \sim D$ and $E \sim G$. From our calculations, we conclude that there is a stable point near the entrance of nanotube.

\section{Summary}

In this paper, we have calculated the total energy of $(10,0)$ CNT incorporated with an $\mathrm{Fe}$ atom by using the $a b$ initio program $\mathrm{DMol}^{3}$. Just below the surface of the nanotube cage, the total energy increases as an Fe atom travels deeper, while on the central axis, the change in the total energy is small near

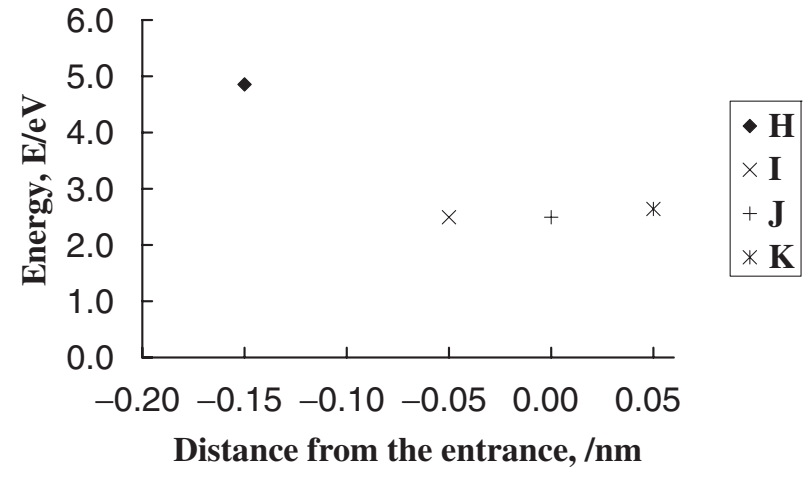

Fig. 9 Result of the total energy at points $H \sim K$ on the center axis.
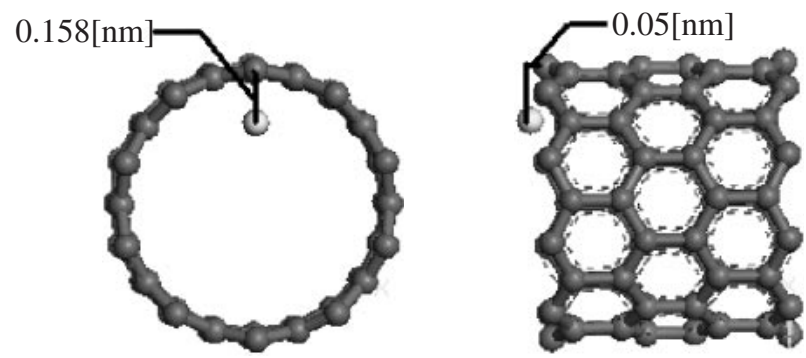

Fig. 10 The most stable position of an Fe atom

the nanotube entrance. When the Fe atom is very close to the nanotube entrance, the difference between the energies inside and outside the nanotube reduces as the Fe atom approaches the central axis. Therefore, we find that the most stable position for the Fe atom is at the point shown in Fig. 10.

According to these results, we find that an $\mathrm{Fe}$ atom does not enter the nanotube automatically. Without any external energy, the $\mathrm{Fe}$ atom will settle at the position shown in Fig. 10. This result agrees with the actual behavior. With regard to the nanotube composition, although $\mathrm{Fe}$ atoms exist around nanocages and fullerenes, they do not enter the SWNTs. This result, that the most stable point is near the nanotube entrance, appears to be related to the fact that the Fe atom acts as a catalyst for SWNT growth.

\section{REFERENCES}

1) H. Kroto, R. Samlley, J. Heath, S. O'Brien and R. Curl: Nature 318 (1985) 162-163.

2) S. Iijima: Nature 352 (1991) 56-58.

3) S. Iijima and T. Ichihara: Nature 363 (1993) 603-605.

4) A. Farajian, K. Ohno, K. Esfarjani, Y. Maruyama and Y. Kawazoe: J. Chem. Phys. 111 (1999) 2164-2168.

5) S. Okada, S. Saito and A. Oshiyama: Phys. Rev. Lett. 86 (2001) 38353838 .

6) E. Hernandez, V. Meunier, B. W. Smith, R. Rurali, H. Terrones, M. Buongiorno Nardelli, M. Terrones, D. E. Luzzi and J. C. Charlier: Nano Letters 3 (2003) 1037-1042.

7) S. Seraphin and D. Zhou: Appl. Phys. Lett. 64 (1997) 2087-2089.

8) B. Delley: J. Chem. Phys. 92 (1990) 508-517.

9) B. Delley: J. Phys. Chem. 100 (1996) 6107-6110.

10) B. Delley: J. Chem. Phys. 113 (2000) 7756-7764.

11) J. P. Perdew and Y. Wang: Phys. Rev. B 45 (1992) 13244-13249.

12) Gaussian 03, M. J. Frisch, et al., Gaussian, Inc., Wallingford CT, 2004. 